\title{
BIOCHEMICAL FAILURE AS SINGLE ABNORMALITY IN PATIENTS WITH PROSTATE CANCER FOLLOWING RADICAL TREATMENT WITH EXTERNAL RADIOTHERAPY: FOLLOW-UP WITHOUT IMMEDIATE TREATMENT
}

\author{
SERGIO L. FARIA, MOHAMUD SALAH ${ }^{1}$, MARC DAVID, LUIS SOUHAMI, MARIE DUCLOS, \\ GEORGE SHENOUDA, FRANÇOIS DEBLOIS, CHRISTIAN JANICK, CAROLYN R. FREEMAN \\ Department of Radio Oncology and ${ }^{1}$ Epidemiology of McGill University, Montreal General Hospital, \\ Montreal, Quebec, Canada
}

\begin{abstract}
Introduction: Biochemical failure has been defined as 3 consecutive increases in PSA following curative treatment of prostate cancer. The appropriate management in such cases is controversial. The most usual treatment has been early introduction of hormones. Such patients will live for many years and hormone therapy causes important secondary effects and increases costs. The guideline in our Department of Radiotherapy has been to follow up, with no initial therapy, cases with low PSA and short PSA doubling time. The present study reports this experience.

Materials and Methods: 528 patients with localized prostate cancer were treated by radical approach between 1992 and 1999, with external radiotherapy, with or without adjuvant hormone therapy. After a median follow-up of 77 months, there were 207 (39\%) cases with biochemical failure, 78 of which were followed without therapy after the identification of biochemical failure. All of them were asymptomatic patients and had negative radiographic examinations or did not have imaging exams requested since they presented a favorable outcome. The follow-up included at least 2 annual visits with physical examination and PSA.

Results: Of the 78 patients with biochemical failure followed without initial therapy, 7 died from other causes than prostate cancer and the remaining 71 cases were alive and asymptomatic in the last follow-up. Prognostic factors previous to radiotherapy such as stage and Gleason score were not considered when deciding for follow-up without initial therapy in these cases. The most significant aspects considered for this decision were low PSA value (median PSA on the last visit for the 78 cases was only $3.9 \mathrm{ng} / \mathrm{mL}$ ) and a slow PSA doubling time (in the present experience the median PSA doubling time was 22.5 months).

Conclusion: There seems to be space for expectant management, without initial hormone therapy, in patients with prostate cancer who present biochemical failure and are asymptomatic after radical external radiotherapy. This decision is important, since early introduction of hormones brings late effects and is expensive. Prospective and randomized studies are required to define this issue.
\end{abstract}

Key words: prostate cancer; radiotherapy; prostate-specific antigen; hormones

Int Braz J Urol. 2004; 30: 289-95

\section{INTRODUCTION}

Prostate cancer is the most common malignant tumor in men in several countries (1). External radiotherapy is a potentially curative alternative and has been largely used for more than 30 years (2). Serum prostate specific antigen (PSA) is a traditional and well-established marker to monitor the follow- 
up of such patients (3). In 1997 the American Society for Therapeutic Radiology and Oncology (ASTRO) published its definition of biochemical failure as being 3 consecutive increases in PSA (4). Though it has not been proved, biochemical failure has been largely used as a marker that anticipates death due to prostate cancer (5). Many patients with prostate cancer, following external radiotherapy, remain asymptomatic, but present a slow increase in PSA as an isolated abnormality. There is no definitive answer about what to do after biochemical failure and thus management in these cases is controversial (6). The most frequently used treatment, even if there is no convincing evidence, has been the introduction of hormones, but there is a concern in relation to costs and side effects (7). Patients with prostate cancer, who are asymptomatic following radiotherapy radical, but with biochemical failure, should live for many years and present clinical progression of cancer only after 8 to 10 years, and eventually die as a consequence of this cancer approximately 12 to 13 years after the diagnosis of biochemical failure (8). This slow evolution has been documented in patients undergoing radical prostatectomy and followed without immediate introduction of therapy following biochemical failure (9).

Initiating the treatment immediately after biochemical failure means that these patients are going to receive hormones for a long time. It is known that long periods of hormone therapy cause significant secondary effects such as loss of libido, impotence, increase in osteoporosis, fractures, and muscle atrophy (8). Not less important, with the exception of orchiectomy, all other alternatives for anti-androgenic treatment are quite expensive. Considering this situation, it is useful to know how patients with biochemical failure evolve when they are uniquely observed without an initial treatment. Our hypothesis is that some patients will die with, but not due to prostate cancer, as in cases of low-risk untreated prostate cancer.

The Department of Radiotherapy, in Montreal, tends to follow asymptomatic cases with biochemical failure, without initial treatment. The present work performs a retrospective assessment of such experience.

\section{MATERIALS AND METHODS}

Between January 1992 and February 1999, 910 cases of prostate cancer were seen in our radiotherapy service, with histological confirmation of adenocarcinoma. Patients included in this review should have localized tumors clinically staged as T1$3 \mathrm{NOM} 0$, have been treated with external radiotherapy (without surgery or brachytherapy), with or without neoadjuvant hormone therapy. These patients should have at least 1 year of follow-up in our service. In accordance to these features, 528 cases were reviewed.

Follow-up was performed at least twice a year, with physical examination and serum PSA. Tomography and bone mapping were required according to clinical need based on judgment by the attending doctor. Patients were treated with a $6 \mathrm{MV}$ - to 18 MV-energy linear accelerator, with 3 or 4 daily fields. Until 1995, planning was traditionally made in 2 dimensions (2D). Since the end of 1995, conformal planning in 3 dimensions (3D) was introduced in the radiotherapy service. The most recent pre-treatment PSA value was considered as the initial PSA value. Overall follow-up time was considered from the date of last radiotherapy application until the last visit or event. The date of biochemical failure was considered as the date in which the first increase in PSA occurred (among 3 consecutive increases). PSA doubling time (or PSADT) was calculated by linear regression considering all PSA values subsequent to the lowest value following radical radiotherapy (called nadir PSA), through mathematical software that automatically finds the curve that better fits PSA values.

Among the 528 patients analyzed, 207 (39\%) had biochemical failure, defined as 3 consecutive increases in PSA separated at least by 4-month interval. When this review was concluded in 2002, of these 207 cases with biochemical failure, 8 did not have data in their records because they had been followed in other institutions, 59 other patients had positive bone mapping and/or pelvic computerized tomography (CT) and received hormone therapy. The remaining 140 patients with biochemical failure were asymptomatic, had negative bone mapping and/or tomography, or these testes were not requested by the attending doctors because they were regarded as 
unnecessary. The decision about initiating treatment for these patients or not was individual, with no specific guideline. It was due to fast increase in PSA, decision by the patient himself, decision by the urologist and/or radiotherapist or case inclusion in clinical trials. Thus, 62 (44\%) of these 140 cases received hormonal treatment following biochemical failure and $78(56 \%)$ were only followed, without receiving any hormonal treatment following biochemical failure. This last group of 78 asymptomatic patients, with biochemical failure and untreated, is the main object of this retrospective analysis.

\section{RESULTS}

Median follow-up times for these 78 patients with biochemical failure and exclusively followed were 77 months. Median age was 70 years (between 49 - 78), median total dose of radiotherapy was 67 Gy (between 61 Gy - 72 Gy), median Gleason score $=6$ (between $2-10), 12$ patients were stage T1, 45 were T2 and 21 were T3. Median initial PSA value = $14 \mathrm{ng} / \mathrm{mL}$ (between 3.1 - 65.0). Median nadir PSA value $=0.4 \mathrm{ng} / \mathrm{mL}$ (between $0-2.8$ ). Median PSA value at the time of diagnosis of biochemical failure was $1.2 \mathrm{ng} / \mathrm{mL}$ (between 0.2 - 20). Median PSA value on the last follow-up was only $3.9 \mathrm{ng} / \mathrm{mL}$ (between 0.7 - 91.1). Median time until the occurrence of biochemical failure was 30 months (between 4 - 72). Median time until PSA nadir value was reached was 12 months. Median follow-up time after biochemical failure was 43 months (between 0 - 112). Finally, median PSA doubling time (PSADT) was 22.5 months (between 6 - 60).

None of these 78 patients died from prostate cancer in the period under evaluation. Seven died from other causes and the remaining 71 were asymptomatic in the last follow-up.

In relation to the initial radiotherapeutic planning, 59\% of cases exclusively followed after biochemical failure were treated with a traditional 2D planning. Table-1 gives a summary of this information and compares data from these 78 untreated patients with those from 62 patients who were treated following biochemical failure. None of these 140 patients died from prostate cancer in the period under evaluation. Comparison between patients, with

Table 1 - Characteristics of 140 among 207 patients with biochemical failure following radical radiotherapy (RT) for localized prostate cancer. All patients were asymptomatic and had negative computerized tomography or bone scintigraphy or did nor undergo any of these testes because they had a highly favorable clinical condition. One group $(n=62)$ received hormone therapy and the other $(n=78)$ was exclusively followed without treatment.

\begin{tabular}{lccc}
\hline Characteristics & Treated $(\mathrm{N}=62)$ & Untreated $(\mathrm{N}=78)$ & $\mathbf{p}^{*}$ \\
\hline Median age & 70 & 70 & 0.82 \\
Median RT dose & $65 \mathrm{~Gy}$ & 6 & 0.08 \\
Gleason score (median) & 6 & 14 & 0.21 \\
Median of initial PSA (ng/mL) & 17 & $\mathrm{~T} 2 \mathrm{~b}$ & 0.69 \\
More frequent stage & $\mathrm{T} 2 \mathrm{~b}$ & $63 \%$ & $0.4(0-2.8)$ \\
Without neoadjuvant hormone therapy & $60 \%$ & $1.2(0.2-20)$ & 0.59 \\
Median nadir PSA after RT & $1.1(0-6.1)$ & $7 / 78$ & $<0.0001$ \\
Median PSA in biochemical failure & $2.2(0.3-15)$ & 77 months \\
Deaths not due to prostate cancer & $2 / 62$ & 31 \\
Median time of overall follow-up & $81 \mathrm{months}$ & 43 \\
Median time until biochemical failure & 23 & 3.4 \\
Median follow-up after biochemical failure & 60 & 12 \\
Median PSA on the last follow-up & 1.8 & 0.0001 \\
Time until nadir was reached (months) & 10 & & 0.36 \\
\hline
\end{tabular}

* Mann-Whitney test; all others were $\chi^{2}$ 
biochemical failure and asymptomatic, both treated and untreated, as summarized in Table-1, is merely an exercise, since patients from the untreated group could have received hormone therapy and vice-versa. Additionally, the process is a dynamic one. Patients without initial treatment can be started on hormone therapy at any time. As shown in Table-1, practically there were no differences between the 2 groups. The few significant differences found suggest that treated patients had a slightly more aggressive PSA kinetics, when compared with untreated patients, with a higher median nadir PSA value $(1.1 \mathrm{ng} / \mathrm{mL}$ versus $0.4 \mathrm{ng} /$ $\mathrm{mL})$, as well as a higher median PSA value at the time of biochemical failure $(2.2 \mathrm{ng} / \mathrm{mL}$ versus $1.2 \mathrm{ng} /$ $\mathrm{mL})$ and a slightly shorter median time until occurrence of biochemical failure (23 versus 31 months).

Since they were given radical radiotherapeutic treatment at the same time, at the same radiotherapy service, remained always asymptomatic and had negative examinations or did not undergo any radiological examination (CT or scintigraphy), we tried to figure out why 62 patients were treated while other 78 cases did not receive any treatment. This decision was not clear in all records, but basically it was determined by the urologist's decision and/or preference of the patients themselves, who were disturbed by seeing their PSA increasing without therapy. Some initiated treatment because they participated in the prospective trial NCIC-PR 7, still ongoing in Canada, aimed to asymptomatic patients with biochemical failure following radical radiotherapy and with PSA greater than $3 \mathrm{ng} / \mathrm{mL}$. The protocol PR 7 randomizes patients comparing immediate intermittent versus continuous hormonal treatment.

\section{COMMENTS}

The present paper reviews 528 cases of patients with localized prostate cancer treated with external radiotherapy, with or without neoadjuvant hormone therapy. Two hundred and seven (39\%) patients had biochemical failure and 78 of these cases were exclusively followed without any treatment. All these cases were asymptomatic and had negative radiographic examinations or were not required to perform imaging tests. During this period, 7 of these patients died from several causes, but not in consequence of prostate cancer. This is the intention of the "watchful waiting" politics. Why were some patients treated while others were not? There is an idiom in the English language that has been used to justify the current indication of hormone therapy for such cases: "chicken switch". The expression refers to the point where patient and doctor are frightened ("chicken") when they see PSA increasing and they activate a device ("switch") in order to decide taking hormones, even if there is no evidence supporting this decision (10).

Some studies have suggested that early hormone therapy can be better than a late approach, but most of this studies included patients with locally advanced or clinically metastatic disease, that form a group that is different from the one featured in the present review. Messing et al., for example, compared initial androgenic treatment versus observation in a small group of 98 cases with prostate cancer following radical prostatectomy and with positive lymph nodes (11). After a median followup of 7.1 years, significantly more men died in the group that was initially observed than in the group with early hormonal treatment. However, the study was terminated before the initially stipulated deadline, there was no central review of the Gleason score (so that there was the possibility of an unequal distribution between both groups) and the cancerspecific survival rate was only $62 \%$ in the observed group, when other contemporary publications reported rates of $80 \%$ in similar situations (12). The English group "MRC Prostate Cancer Working Party Investigators" compared, in a prospective and randomized manner, the effect of early versus late endocrine therapy also in patients with locally advanced extensive disease, or clinically metastatic disease, but with no symptoms (13). 934 patients took part in this study. The follow-up method and subsequent therapy were not standardized. They were decided upon the usual practice of each participating doctor. As a result, a significant number of patients with late therapy had more pathological fractures and other symptoms, compared with 
patients that received early hormone therapy. However, some patients from "late" group never received hormonal treatment in any moment, suggesting that the treatment was initiated too late or it was never offered. These 2 studies have been also referred in an attempt to warrant immediate hormonal treatment following biochemical failure also for asymptomatic patients, after radical radiotherapeutic treatment. We understand that, in addition to methodological criticism, these are completely different clinical situations.

In the present study, which assessed biochemical failure following radiotherapy, the 78 patients were asymptomatic, had no metastases and presented as their only abnormality a slow increase in serum PSA, forming, thus, a selected population that is different from the 2 studies previously mentioned. In our Department of Radiotherapy, there is a trend towards the non-introduction of initial treatment in cases that present low PSA and a slow increase over time, that is, a long PSADT time. Hormone therapy has been initiated basically when PSA values exceed 10 or $20 \mathrm{ng} / \mathrm{mL}$ or PSADT $>8$ months. In the present experience, after 77 months of follow-up, median PSA in the last visit of 78 cases followed without therapy was only $3.9 \mathrm{ng} / \mathrm{mL}$. Prognostic tables for prostate cancer include PSA values $<10 \mathrm{ng} / \mathrm{mL}$ or $20 \mathrm{ng} / \mathrm{mL}$ as a good prognostic factor (14) and consequently these have been our reference values for initiating therapy after biochemical failure.

In the present review, median PSADT time of the 78 patients that were followed without treatment was 22.5 months, reflecting the selection of cases with slow tumoral evolution. The group from MD Anderson hospital, in Houston, has several publications concerning PSA kinetics in patients with prostate cancer following radical external radiotherapy (15). They conclude that the metastasis rate significantly ranges depending on PSADT. Actuarial metastasis rates in 7 years were $54 \%$ and $7 \%$, for cases with PSADT $>8$ months and PSADT $<8$ months, respectively.

Patients with newly diagnosed prostate cancer can be exclusively followed without initial therapy ("watchful waiting") with good results for cases with low risk or early stages $(16,17)$. In the present experience, initial prognostic factors such as Gleason score or staging $(\mathrm{T})$, were not considered for deciding whether initiate hormone therapy or not after biochemical failure. Thus, there were more T3 ( 21 cases) than $\mathrm{T} 1$ cases ( 12 cases) in the group of 78 patients who were exclusively followed. Similarly there were patients with all levels of Gleason score, ranging from 2 to 10 .

Finally, the request of radiographic examinations for assessing metastases, particularly bone scintigraphy, in asymptomatic patients with biochemical failure, is not well defined and varies among institutions and even among their professionals. The probability of a positive bone scintigraphy is close to zero in patients with PSA $<15 \mathrm{ng} / \mathrm{mL}$ (18). Recent guides ("guidelines") for prostate cancer (19) suggest that in patients who are not under anti-androgenic therapy, the risk of positive bone scintigraphy is lower than $5 \%$ of cases when PSA is lower than $40-50 \mathrm{ng} / \mathrm{mL}$, and consequently this examination should not be routinely requested in asymptomatic patients with low PSA. More than $1 / 3$ of our patients with biochemical failure were not requested to perform bone scintigraphy because they were asymptomatic and had low PSA value.

\section{CONCLUSION}

This retrospective analysis found 207 (39\%) out of 528 patients with localized prostate cancer and treated with radical radiotherapy, between 1992 and 1999, with biochemical recurrence after 77 months of median follow-up. There is a lot of controversy about what to do, and when, after biochemical failure. The most common treatment has been the introduction of early anti-androgenic therapy, though there is no evidence that this approach offers better survival than a slightly later treatment.

Our hypothesis is that asymptomatic patients with low PSA and long PSADT after biochemical failure can undergo an expectant management in a first moment, without worsening the final outcome. The present retrospective analysis showed that among the 78 (39\%) patients who were exclusively followed 
without any therapy following biochemical failure, there was no death from prostate cancer or metastasis, suggesting that there is room for delay when initiating the treatment in selected cases. Patients were not treated because they had low PSA level (in general PSA $<10 \mathrm{ng} / \mathrm{mL}$ ) and long PSADT (in general $>8$ months) during follow-up, without major concern with early prognostic factors such as staging or Gleason score.

Selecting patients with biochemical failure so they can be exclusively followed, and initiating hormone therapy only when the tumor shows signs of higher aggressiveness, is the most cost-effective alternative that should offer better quality of life, since these patients will not suffer the secondary effects caused by anti-androgenic therapy over long periods. As usual, the only way to know if there is a difference between early or late hormonal therapy in this particular group of patients, is through prospective and randomized studies. This concept has been previously published (20). Our suggestion, for an eventual study in Canada, is to compare immediate hormone therapy versus a selective delay. In the latter case, asymptomatic patients with biochemical failure would initiate treatment only with PSA $>10 \mathrm{ng} / \mathrm{mL}$ and PSADT $<8$ months, or upon clinical progression of the tumor, confirmed by some radiological examination.

\section{REFERENCES}

1. INCA - Ministério da Saúde: Mortalidade por Câncer. www.inca.gov.br. Rio de Janeiro, Brazil, 2002.

2. Ray GR, Cassady JR, Bagshaw MA: Definitive radiation therapy of carcinoma of the prostate. A report on15 years experience. Radiology. 1973; 106: 40718.

3. Polascik TJ, Oesterling JE, Partin AW: Prostate specific antigen: a decade of discovery — what we have learned and where we are going. J Urol. 1999; 162: 293-306.

4. ASTRO Consensus Panel: Guidelines for PSA following RT: Int J Radiat OncolBiol Phys. 1997; 37 : 1035.

5. D'Amico AV, Cote K, Loffredo M, Renshaw AA, Chen $\mathrm{MH}$ : Pretreatment predictors of time to cancer specific death after PSA failure. J Urol. 2003; 169: 1320-4.
6. Duchesne GM, Millar JL, Moraga V, Rosenthal M, Royce P, Snow R: What to do for prostate cancer patients with a rising PSA? A survey of Australian practice. Int J Radiat Oncol Biol Phys. 2003; 55: 98691.

7. Herr HW, O'Sullivan M: Quality Of Life of asymptomatic men with nonmetastatic prostate cancer on androgen deprivation therapy. J Urol. 2000; 163: 1743-6.

8. Schroder FH: Endocrine treatment of prostate cancer. BJU Int. 1999; 83: 161-70.

9. Pound CR, Partin AW, Eisenberger MA, Chan DW, Pearson JD, Walsh PC: Natural history of progression after PSA elevation following radical prostatectomy. JAMA. 1999; 281: 1591-7.

10. Johnstone PA: Biochemical failure after definitive therapy of prostate cancer. Defining the "chicken switch". Int J Radiat Oncol Biol Phys. 2003; 55: 85960.

11. Messing EM, Manola J, Sarosdy M, Wilding G, Crawford ED, Trump D: Immediate hormonal therapy compared with observation after radical prostatectomy and pelvic lymphadenectomy in men with node positive prostate cancer. N Eng J Med. 1999; 341: 1781-8.

12. Eisenberger MA, Walsh PC: Early androgen deprivation for prostate cancer? Editorial, N Eng J Med. 1999; 341: 1837-8.

13. Medical Research Council Prostate Working Party Investigators Group: Immediate vs deferred treatment for advanced prostate cancer. Br J Urol. 1997; 79: 23546.

14. Partin AW, Kattan MW, Subong EN, Walsh PC, Wojno $\mathrm{KJ}$, Oesterling JE, et al.: Combination of prostatespecific antigen, clinical stage, and Gleason score to predict pathological stage of localized prostate cancer. A multi-institutional update. JAMA. 1997; 277: 144551.

15. Zagars GK, Pollack A: Kinetics of serum PSA after external beam radiation for clinically localized prostate cancer. Radiother Oncol. 1997; 44: 213-21.

16. Chodak GW, Thisted RA, Gerber GS: Results of conservative management of clinically localized prostate cancer. N Engl J Med. 1994; 330: 242-8.

17. Klotz LH, Choo R, Morton G, Danjoux C: Expectant management with selective delayed intervention for favorable risk prostate cancer. Can J Urol. 2002; 9 (suppl 1): 2-7.

18. Yap BK, Choo R, Deboer G, Klotz L, Danjoux C, Morton G.: Are serial bone scans useful for the follow- 
up of clinically localized, low to intermediate grade prostate cancer managed with watchful observation alone? BJU Int. 2003; 91: 613-7.

19. Scherr D, Swindle PW, Scardino P: National Comprehensive Cancer Network Guidelines for the
Management of Prostate Cancer. Urology. 2003; 61 (2 suppl 1): 14-24.

20. Parker C, Dearnaley DP.: The management of PSA failure after radical radiotherapy for localized prostate cancer. Radiother Oncol. 1998; 49: 103-10.

Received: February 2, 2004

Accepted after revision: June 6, 2004

\author{
Correspondence address: \\ Dr. Sérgio Luiz Faria \\ Department of Radiation Oncology \\ Montreal General Hospital \\ 1650 Cedar Av. \\ H3G 1A4 Montreal, Quebec, Canada \\ Phone: + 514 934-8040 \\ E-mail: sergio.faria@muhc.mcgill.ca
}

\section{EDITORIAL COMMENT}

Biochemical recurrence following monotherapy for prostate cancer with external radiotherapy is a problem commonly found by the urologist in daily practice and its management is not always easy, since there is controversy in the literature about what should be done in such situation. The institution of adjuvant hormone therapy (continuous or intermittent) is associated with high costs and several changes that interfere in the patient's quality of life, such as loss of libido, impotence, depression, osteoporosis and loss of muscular mass.

Thus, the authors report the experience with a recent series of patients treated by external radiotherapy, with or without neoadjuvant hormone therapy, where biochemical recurrence following treatment was observed in $39 \%$ of cases. Among the asymptomatic cases with no clinical, tomographic or scintigraphic evidence of progressive disease, the decision of initiating adjuvant hormone therapy was individual, but one group of 78 selected patients, based on the absence of clinical evidence of disease and slow increase in PSA (long doubling time), was followed without receiving adjuvant hormone therapy. The results in this group (with a median of 77 months of follow-up) were excellent, with no case of clinical metastasis or specific mortality to the moment. In the described series, the value of nadir PSA, the PSA upon detection of biochemical failure and the mean time until the occurrence of biochemical failure seem to have been determinant for selecting the group that was treated exclusively with observation. The paper strongly demonstrates that there is really room for careful observation of a selected group of patients with post-radiotherapy biochemical recurrence, especially if they do not present clinical or radiological signs of progression, and if the PSA doubling time is long (> 8 months). However, absolute results concerning these issues will be revealed only after the conclusion of prospective and randomized studies, as the authors personally stress.

Dr. Gustavo Franco Carvalhal Associate Professor, Catholic University Porto Alegre, RS, Brazil 